\title{
On the Relationship Between Non-alcoholic Fatty Liver Disease with Body Composition and Bone Mineral Density in Overweight/Obese Adolescents
}

\author{
Zahra Dehnavi ${ }^{1}$, Hanieh Barghchi ${ }^{1}$, Fatemeh Roudi (iD ${ }^{1}$, Mahmoud Belghaisi Naseri ${ }^{1}$, Andisheh \\ Nourozian Ostad ${ }^{1}$, Zahra Khorasanchi ${ }^{1}$, Mohsen Nematy ${ }^{2}$ and Farkhondeh Razmpour ${ }^{3,}$ \\ ${ }^{1}$ Department of Nutrition, Faculty of Medicine, Mashhad University of Medical Sciences, Mashhad, Iran \\ ${ }^{2}$ Metabolic Syndrome Research Center, Department of Nutrition, Faculty of Medicine, Mashhad University of Medical Sciences, Mashhad, Iran \\ ${ }^{3}$ Department of Nutrition, Faculty of Medicine, Bandar Abbas University of Medical Sciences, Bandar Abbas, Iran \\ "Corresponding author: Department of Nutrition, Faculty of Medicine, Bandar Abbas University of Medical Sciences, Bandar Abbas, Iran. Tel: +98-7633424175, Fax: \\ +98-7632254455, Email: frazmpoor@gmail.com
}

Received 2021 January 20; Revised 2021 March 14; Accepted 2021 April 05.

\begin{abstract}
Background: Nonalcoholic fatty liver disease (NAFLD) is a health problem growing in line with the rising prevalence of obesity in children and adolescents, which may be correlated with different metabolic abnormalities such as osteoporosis.

Objectives: This study aimed to evaluate the possible relationship between NAFLD with body composition and bone mineral density (BMD) in obese and overweight adolescents.

Methods: This cross-sectional study encompassed 70 adolescents aged 11 - 18 years and was conducted during March 2016 and September 2016 in Mashhad, Iran. Anthropometric parameters and blood biomarkers were measured. Fat mass, fat-free mass, and BMD were determined using dual-energy X-ray absorptiometry (DXA) scans, and NAFLD was also assessed using Fibroscan. All statistical data were analyzed using SPSS software version 21. Multivariate linear regression assessed the relationship between liver fat content with bone-related indicators, and multivariate logistic regression detected the relationship between body composition and NAFLD.

Results: Total and trunk fat mass were significantly correlated with higher NAFLD even after controlling for intervening factors (total fat mass, $\mathrm{OR}=1.27 ; 95 \% \mathrm{CI}, 1.016$ to $1.59, \mathrm{P}=0.036$; trunk fat mass, $\mathrm{OR}=1.35 ; 95 \% \mathrm{CI}, 0.97$ to $1.88, \mathrm{P}=0.045$ ). Moreover, liver fat content was significantly correlated with lower BMD Z-score after adjusting for gender, BMI Z-score, ALT, fat mass index, total lean mass, and physical activity $(\beta=-0.285, \mathrm{P}=0.048)$.

Conclusions: The findings of the present study suggest that excess adipose tissue is correlated with higher NAFLD. Moreover, liver steatosis may be correlated with decreased BMD Z-score in overweight/obese adolescents.
\end{abstract}

Keywords: Nonalcoholic Fatty Liver Disease, Adolescent, Body Composition, Bone Density, Osteoporosis

\section{Background}

Childhood obesity, a serious worldwide growing health problem, is considered as one of the primary etiologies of nonalcoholic fatty liver disease (NAFLD) and other chronic diseases in childhood (1-4). According to previous studies, up to $33 \%$ of obese children suffer from NAFLD (5), which may also be associated with an increased risk of type 2 diabetes, cardiovascular diseases, liver fibrosis, hepatic cirrhosis, liver failure, and hepatocellular carcinoma (6-8). NAFLD is a multifactorial disorder in which both genome and environmental factors are involved (9). Notably, the distribution of excessive adipose tissue and total fat plays a critical role in obesity-induced NAFLD and its relevant comorbidities (10).

On the other hand, osteoporosis is considered a major fall-related death etiology mostly observed in the elderly (11). An optimal peak bone mass achievement in early adulthood is believed to be the best protective factor against future osteoporosis (12). According to the literature, the childhood era, especially the peri-pubertal stage, is a critical period for bone mass accretion $(12,13)$. In addition to the obesity-related lifestyle factors such as sedentary lifestyles, hepatic steatosis/NAFLD may be associated with decreased bone mineral density (BMD) (14). This is, while this field of research has been disregarded in the literature. 


\section{Objectives}

The present study follows two primary objectives: (1) to examine the possible relationship between NAFLD and body composition, especially the distribution of body fat; (2) to investigate the probable relationship between NAFLD with BMD in overweight/obese adolescents.

\section{Methods}

\subsection{Study Population}

This cross-sectional study was carried out during March- September 2016 in Mashhad, Iran. The participants were non-randomly selected from the overweight and obese children admitted to a nutrition clinic.

\subsubsection{Inclusion Criteria}

Adolescents aged 11 - 18 years and BMI is $>$ 85th percentile. The exclusion criteria of the study were suffering from chronic diseases (e.g., viral hepatitis, autoimmune/congenital liver disease, congenital metabolic diseases, and cancer) and undergoing any type of surgery during the last six months. Accordingly, 70 overweight and obese adolescents ( 37 boys and 33 girls) participated in this study. Written informed consent forms were provided by all the parents or the legal guardians of the participants. The study was approved by the Ethics Committee at Mashhad University of Medical Sciences (MUMS) (ethical code: IR.MUMS.fm.REC.1395.64).

\subsection{Sample Size}

The present study is a part of our previous study entitled "plasma levels of vascular endothelial growth factor and its soluble receptor in nonalcoholic fatty liver disease" (15) and published in 2018. Since our previous project was partly a student dissertation and partly a university project, we had to publish the findings in two papers. In the primary project, the sample size was determined using the following formula:

$N=\left(Z 1-\frac{\alpha}{2}+Z 1-\beta\right) \frac{S 1+S 2}{X 1-X 2}$

Where, type1 error $(\alpha)=0.05$; type 2 error $(\beta)=80 \%$ (power); $\mathrm{X} 1=$ mean in group $1 ; \mathrm{X} 2=$ mean in group $2 ; \mathrm{Z1}=$ standard deviation in group $1 ; \mathrm{Z} 2=$ standard deviation in group 2.

Accordingly, the sample size was estimated to be 70 .

\subsection{Data Collection}

Anthropometric parameters, including weight $(\mathrm{kg})$, height $(\mathrm{cm})$, and waist circumference $(\mathrm{cm})$, were determined based on standard protocols. Body height was measured to the nearest $0.01 \mathrm{~cm}$ with barefoot in a standing position, using a standard stadiometer. Bodyweight was measured by a clinical scale (SECA) to the nearest 0.1 kilograms. Waist circumference was measured in the horizontal plane midway between the lower ribs and the iliac crest. Body mass index (BMI) was defined as an individual's body weight divided by the square of his or her height (standard unit of measure is $\mathrm{kg} / \mathrm{m}^{2}$ ).

The participants' physical activity was assessed using the Baecke Habitual Physical Activity Questionnaire (BH$\mathrm{PAQ})$. The questionnaire evaluates individuals' habitual physical activity on a scale ranging from 1 to $5(16,17)$, with a score of 1 and 5 indicating the lowest and highest levels of activity (18). The reliability and validity of BHPAQ (Farsi version) are already confirmed (19).

Blood samples were obtained from the participants' antecubital veins after 10 - 12-hour fasting to determine the levels of fasting blood glucose (FBG), fasting insulin (FI), hemoglobin A1c, 25(OH) Vitamin D, and full-fasting lipid profile, including the levels of triglyceride (TG), total cholesterol (TC), low-density lipoprotein cholesterol (LDLc), and high-density lipoprotein cholesterol (HDL-c) using standard automated laboratory techniques. Moreover, liver function enzymes, including aspartate aminotransferase (AST), alanine aminotransferase (ALT), and gammaglutamyl transferase (GGT), were measured as well.

\subsection{Measurement of Fat Mass, Fat-Free Mass, and Bone Mineral Density Using DXA}

Whole-body composition, including bone mineral density (BMD), bone mineral content (BMC), lean mass (LM), and fat mass (FM), was determined using whole-body dual-energy X-ray absorptiometry (DXA) scans (GE Lunar Corp, Madison, WI, USA) at the radiology department of Imam Reza Hospital, Mashhad University of Medical Sciences, Mashhad, Iran. All DXA scans and subsequent insoftware analyses were completed by the same certified radiological technologist blinded to the participants' characteristics. The DXA scan machine was calibrated in daily examinations based on the manufacturer's guidelines.

All the scan results were divided into total and regional FM, LM, BMD, and BMC scans. Moreover, two android and gynoid regions were measured using DXA scan. Furthermore, adipose indices were calculated as follows: (1) A/G ratio [the ratio of android $(\mathrm{kg})$ divided by gynoid $(\mathrm{kg})],(2)$ fat mass index (FMI) [fat mass/height $\left.{ }^{2}\left(\mathrm{~kg} / \mathrm{m}^{2}\right)\right]$, (3) \%fat trunk/ $\%$ fat legs, and (4) trunk/limb fat mass ratio. Lean + BMC indices were also calculated as follows: (1) lean mass index 
(LMI) (lean $+\mathrm{BMC} /$ height $\left.^{2}\right)$, and (2) appendicular lean mass index (ALMI) (appendicular lean mass $+\mathrm{BMC} /$ height $^{2}$ ).

\subsection{Measurement of Liver Steatosis Using Fibroscan}

Fibroscan approach was used to assess liver steatosis and fibrosis (Echosense504), in which liver stiffness was measured using shear wave velocity (20). Controlled attenuation parameter (CAP) is a novel noninvasive tool using ultrasound attenuation for liver steatosis measurement, which has been implemented by the Fibroscan approach (21).

Fibroscan was performed on a standardized machine (Echosens, Paris, France) by a certified operator utilizing a small $(\mathrm{S})$ or medium $(\mathrm{M})$ probe to assess both $\mathrm{CAP}(\mathrm{dB} / \mathrm{m})$ and liver stiffness examinations (LSM) (kPa) simultaneously. A reliable LSM was defined as the median value of 10 measurements (a success rate of $>60 \%$, and an IQR $<30 \%$ of the median LSM value)(22). The severity of liver steatosis was assessed according to the following cutoffs for CAP: (1) $<225 \mathrm{db} / \mathrm{m}$ (no steatosis), (2) $225-250 \mathrm{db} / \mathrm{m}$ (mild steatosis), (3) $250-300 \mathrm{db} / \mathrm{m}$ (moderate steatosis), (4)> $300 \mathrm{db} / \mathrm{m}$ (severe steatosis) (23). Moreover, CAP $<225 \mathrm{db} / \mathrm{m}$ as and CAP $>225 \mathrm{db} / \mathrm{m}$ were set as no NAFLD and NAFLD, respectively.

\subsection{Statistical Analysis}

All the statistical analyses were conducted using SPSS software version 21. The Kolmogorov-Smirnov test was used to examine the data normality. Normally distributed quantitative data were described by mean and standard deviation, and non-parametric data were described using median and interquartile range (IQR). Furthermore, qualitative data were described as frequency and percentage. Independent-sample $t$-test and Mann-Whitney test were used to compare continuous variables in the two groups, and a chi-square test was conducted for categorical variables.

The relationship between liver fat content with BMD, BMD Z-score, and BMC were evaluated using multivariate linear regression analysis. Standardized $\beta$ was represented as the coefficient $\beta$. The relationship between different parts of body composition and NAFLD were assessed using multivariate logistic regression analysis, and the odds ratios (OR) and 95\% confidence intervals (CI) were calculated. $\mathrm{P}<0.05$ was set as the significance level in all tests.

\section{Results}

Table 1 shows the participants' general specifications. The study participants' mean \pm SD age $(n=70)$ was 12.78 \pm 2.91 years, and $52.8 \%$ of the participants were male. The mean \pm SD of BMI and waist circumference were $28.14 \pm$ $4.91 \mathrm{~kg} / \mathrm{m}^{2}$ and $91.34 \pm 11.52 \mathrm{~cm}$, respectively. Forty-four adolescents $(62.8 \%)$ with NAFLD had significantly higher height, weight, BMI, BMI Z-score, and waist circumference, in comparison to adolescents with no NAFLD $(\mathrm{P}<0.05)$. Nonetheless, no significant difference was observed between the adolescents with NAFLD or with no NAFLD in terms of age, level of physical activity (baecke score), and all the laboratory variables $(P>0.05)$, except for fasting in$\operatorname{sulin}(\mathrm{P}=0.024)$.

Among fat mass indices assessed by DXA scan, total and trunk FM, android and gynoid FM, and FMI were significantly higher in adolescents with NAFLD than adolescents with no NAFLD $(P<0.05)$ (Table 2). Moreover, NAFLDpositive adolescents had higher mean total LM, total (LM + BMC), LMI, ALMI, and total mass than adolescents with No $\operatorname{NAFLD}(\mathrm{P}<0.05)$ (Table 2).

BMC at the trunk $(\mathrm{P}=0.017)$ and whole-body $(\mathrm{P}=0.012)$ were significantly higher in NAFLD positive group than adolescents with no NAFLD; however, there was no significant difference between the two groups in terms of total BMD $(\mathrm{P}=0.198)$, total BMD Z-score $(\mathrm{P}=0.093)$, and BMD at thoracic spine $(\mathrm{P}=0.097)$.

Table 3 demonstrates the relationships between fat and fat-free mass patterning indices and NAFLD in overweight/obese adolescents using multivariable regression analyses. Independent of age, higher total FM was significantly correlated with increased NAFLD BMI Z-score (model $2, \mathrm{OR}=1.33, \mathrm{P}=0.003$ ), ALT, T-cholesterol (model $3, \mathrm{OR}=1.33$, $\mathrm{P}=0.007$ ), fasting insulin, and physical activity (model 4 , $\mathrm{OR}=1.27, \mathrm{P}=0.036$ ). The relationship between trunk FM and NAFLD revealed the same pattern. Moreover, higher android and gynoid FM and FMI were significantly correlated with increased NAFLD in the crude model (model 1) and model $2(\mathrm{P}<0.05)$. In this regard, this relationship attenuated to the null value when we additionally controlled for ALT, TC, fasting insulin, and physical activity (models 3 and 4$)(P>0.05)$. There was no significant relationship between total LM and ALMI with NAFLD after controlling for intervening factors in the adjusted models $(\mathrm{P}>0.05)$.

Multivariate linear regression analyses were conducted to investigate the relationship between liver fat content (\%) with BMD and BMC (Table 4). There was no significant relationship between total BMD and liver fat content neither in the crude model nor other adjusted models $(\mathrm{P}>0.05)$. Regarding total BMD Z-score, there was no significant relationship between liver fat content (\%) and total BMD Z-score in the crude model $(\beta=-0.205, \mathrm{P}$ $=0.094)$, model $2(\beta=-0.217, \mathrm{P}=0.105)$, and model $3(\beta$ $=-0.263, \mathrm{P}=0.07)$; however, when total LM and physical activity were interned additionally into the model 4 , a significant relationship was noticed between liver fat 


\begin{tabular}{|c|c|c|c|}
\hline Variables & No $\operatorname{NAFLD}(N=26)$ & $\operatorname{NAFLD}(N=44)$ & P Value \\
\hline Gender & & & 0.9 \\
\hline Women & $14(36.4)$ & $23(63.6)$ & \\
\hline Men & $12(37.8)$ & $21(62.2)$ & \\
\hline Age $(y)$ & $11.70 \pm 0.55$ & $13.47 \pm 0.42$ & 0.08 \\
\hline Diabetes Mellitus & $0(0.0)$ & $3(100)$ & 0.289 \\
\hline Dyslipidemia & $1(33.3)$ & $2(66.6)$ & 0.999 \\
\hline Height $(\mathrm{cm})$ & $148.15 \pm 13.74$ & $158.11 \pm 11.30$ & 0.002 \\
\hline Weight (kg) & $56.56 \pm 14.71$ & $74.87 \pm 19.28$ & 0.000 \\
\hline $\operatorname{BMI}\left(\mathrm{kg} / \mathrm{m}^{2}\right)$ & $25.43 \pm 3.53$ & $29.60 \pm 5.23$ & 0.001 \\
\hline BMI Z-score & $1.72 \pm 0.45$ & $1.95 \pm 0.37$ & 0.028 \\
\hline $\mathrm{WC}(\mathrm{cm})$ & $85.30 \pm 10.06$ & $95.14 \pm 12.08$ & 0.001 \\
\hline Liver stiffness value (kPa) & $5.11 \pm 0.22$ & $5.27 \pm 0.20$ & 0.715 \\
\hline $\mathbf{C A P}(\mathbf{d B} / \mathbf{m})$ & $215.88 \pm 21.42$ & $284.27 \pm 28.14$ & 0.000 \\
\hline Liver fat content (\%) & $8.19 \pm 0.38$ & $52.69 \pm 3.26$ & 0.000 \\
\hline Physical activity (Baecke score) & $5.65 \pm 0.15$ & $5.58 \pm 0.11$ & 0.503 \\
\hline FBS (mg/dL) & $84.65 \pm 7.71$ & $83.68 \pm 9.32$ & 0.655 \\
\hline HbA1C & $5.12 \pm 0.15$ & $5.14 \pm 0.12$ & 0.661 \\
\hline $\operatorname{AST}(\mathbf{I U} / \mathbf{L})$ & $24.19 \pm 1.28$ & $24.21 \pm 2.42$ & 0.298 \\
\hline $\operatorname{ALT}(\mathbf{I U} / \mathbf{L})$ & $23.80 \pm 2.32$ & $30.45 \pm 4.78$ & 0.173 \\
\hline GGT (mg/dL) & $20.76 \pm 1.29$ & $22.85 \pm 1.50$ & 0.443 \\
\hline TG $(\mathbf{m g} / \mathbf{d L})$ & $97.19 \pm 6.97$ & $100.31 \pm 5.26$ & 0.679 \\
\hline $\mathrm{TC}(\mathrm{mg} / \mathrm{dL})$ & $150.92 \pm 25.14$ & $144.09 \pm 23.25$ & 0.253 \\
\hline LDL-c (mg/dL) & $82.42 \pm 14.99$ & $78.25 \pm 18.07$ & 0.325 \\
\hline HDL-c (mg/dL) & $39.27 \pm 1.49$ & $39.69 \pm 1.14$ & 0.765 \\
\hline $25(\mathrm{OH})$ vitamin $\mathrm{D}(\mathrm{ng} / \mathrm{mL})$ & $29.28 \pm 4.06$ & $31.00 \pm 2.93$ & 0.894 \\
\hline Fasting insulin $(\mu \mathrm{IU} / \mathbf{m L})$ & $8.84 \pm 1.01$ & $13.76 \pm 1.41$ & 0.024 \\
\hline
\end{tabular}

Abbreviations: NAFLD, nonalcoholic fatty liver disease; BMI, body mass index; WC, waist circumference; FBS, fasting blood sugar; HBA1C, hemoglobin A1C; AST, aspartate aminotransferase; ALT, alanine aminotransferase; GGT, gamma-glutamyl transferase; TG, triglycerides; TC, total cholesterol; LDL, low-density lipoprotein; HDL, highdensity lipoprotein.

a Values are indicated as mean \pm SD and No. (\%) unless otherwise indicated.

${ }^{b}$ Differences between children with and without NAFLD.

${ }^{\mathrm{c}}$ Values are significant at $\mathrm{P}<0.05$.

content (\%) and lower BMD Z-score $(\beta=-0.285, \mathrm{P}=0.048)$. Liver fat content was not significantly associated with Total BMC after controlling for intervening factors in the adjusted models $(\mathrm{P}>0.05)$.

\section{Discussion}

The findings of the present study indicate that fat massrelated indices, especially total and trunk fat mass, are independently correlated with increased NAFLD, suggesting that excess body fat may play a crucial role in the pathogenesis of fatty liver in overweight/obese children and adoles- cents. In line with the findings of the present study, several studies have reported the relationship between body fat mass and NAFLD in adults and children (24-27). According to Monteiro et al., trunk fat mass and intra-abdominal adipose tissue were the significant predictors of NAFLD in obese children and adolescents (25). Furthermore, in our previous study, waist circumference and BMI were positively correlated with liver steatosis and fibrosis in children and adolescents, thereby predicting NAFLD (28). Similarly, Yang et al. reported the relationship between body fat mass with insulin resistance and subsequent NAFLD in obese children (29). 


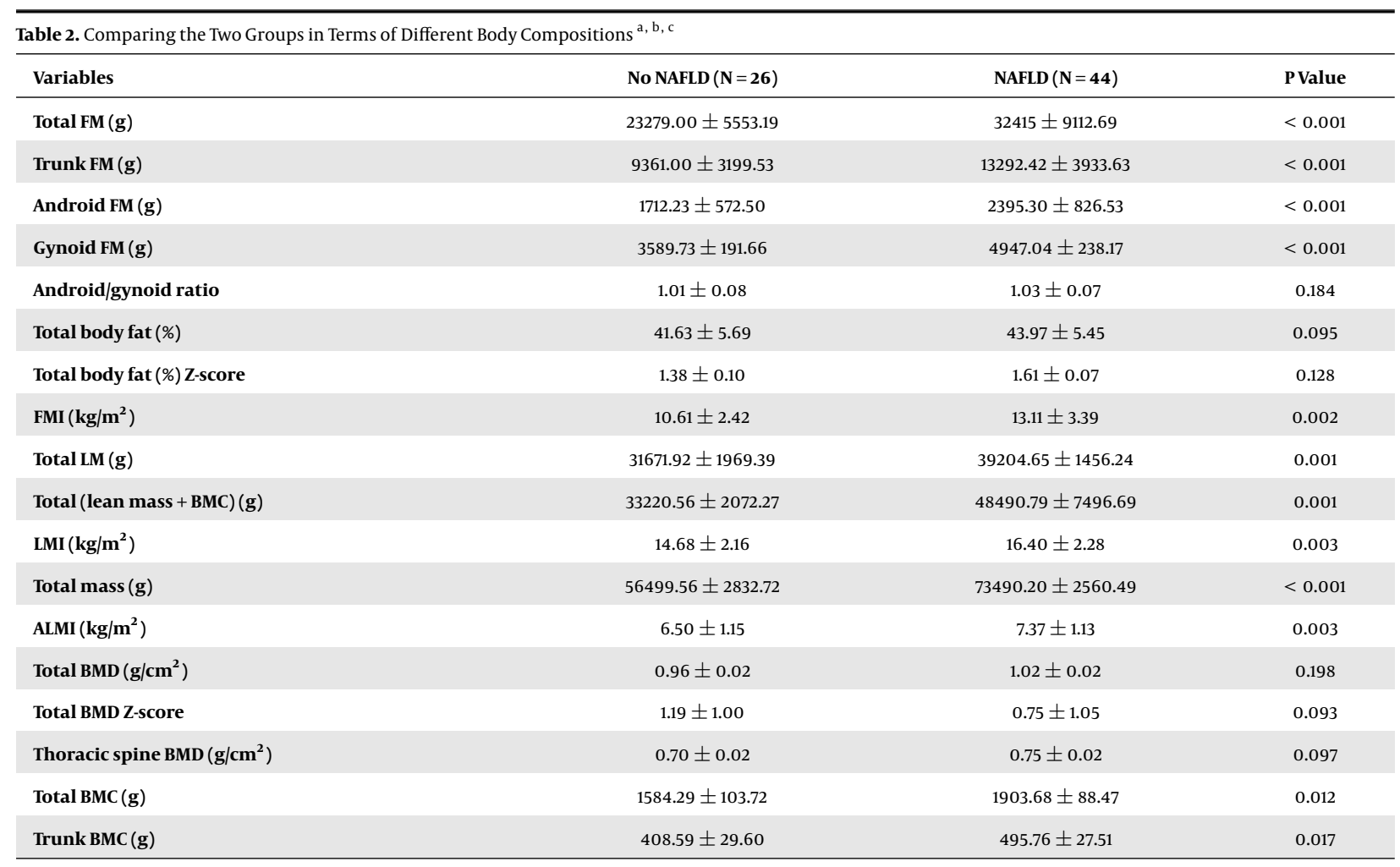

Abbreviations: NAFLD, nonalcoholic fatty liver disease; BMC, bone mineral content; BMD, bone mineral density; FM, fat mass; FMI, fat mass index; LM, lean mass; LMI, lean mass index; ALMI, appendicular lean mass index.

${ }^{a}$ Values are indicated as mean \pm SD unless otherwise indicated.

${ }^{\mathrm{b}}$ Differences between children with and without NAFLD

${ }^{c}$ Values are significant at $\mathrm{P}<0.05$.

\begin{tabular}{|c|c|c|c|c|c|c|c|c|}
\hline \multirow{2}{*}{ Variables } & \multicolumn{2}{|c|}{ Model1 } & \multicolumn{2}{|c|}{ Model 2} & \multicolumn{2}{|c|}{ Model 3} & \multicolumn{2}{|c|}{ Model 4} \\
\hline & OR $(95 \% \mathrm{CI})$ & PValue & OR $(95 \% \mathrm{CI})$ & PValue & OR $(95 \% \mathrm{CI})$ & PValue & $\mathrm{OR}(95 \% \mathrm{CI})$ & P Value \\
\hline Total FM & $1.24(1.11-1.39)$ & $<0.001$ & $1.33(1.1-1.6)$ & 0.003 & $1.33(1.08-1.63)$ & 0.007 & $1.27(1.016-1.59)$ & 0.036 \\
\hline Trunk FM & $1.51(1.2-1.9)$ & $<0.001$ & $\begin{array}{c}1.54 \\
(1.086-2.19)\end{array}$ & 0.015 & $1.5(1.046-2.15)$ & 0.03 & $1.35(0.97-1.88)$ & 0.045 \\
\hline Android FM & $6.26(2.2-17.8)$ & 0.001 & $\begin{array}{c}5.24 \\
(0.89-30.6)\end{array}$ & 0.048 & $4.12(0.62-27.1)$ & 0.14 & $\begin{array}{c}2.52 \\
(0.45-14.14)\end{array}$ & 0.29 \\
\hline Gynoid FM & $2.82(1.57-5.03)$ & $<0.001$ & $2.35(1.12-4.92)$ & 0.02 & $2.1(0.94-4.7)$ & 0.07 & $1.8(0.82-3.94)$ & 0.14 \\
\hline FMI & $1.45(1.15-1.84)$ & 0.002 & $1.36(0.99-1.87)$ & 0.049 & $1.41(0.96-2.06)$ & 0.075 & $1.3(0.87-1.97)$ & 0.19 \\
\hline Total LM & $1.10(1.033-1.17)$ & 0.003 & $1.17(0.82-1.66)$ & 0.38 & $1.02(0.91-1.15)$ & 0.67 & $\begin{array}{c}1.003 \\
(0.89-1.12)\end{array}$ & 0.96 \\
\hline
\end{tabular}

Abbreviations: NAFLD, nonalcoholic fatty liver disease; FM, fat mass; FMI, fat mass index; LM, lean mass; ALMI appendicular lean mass index; OR, odds ratio; CI, confidence interval; BMI, body mass index; ALT, alanine aminotransferase; T-cholesterol, total cholesterol.

a Model 1, crude; Model 2, adjusted with age and BMI Z-score; Model 3, model 2 was additionally adjusted with ALT and T-cholesterol; Model 4, model 3 was additionally adjusted with fasting insulin and physical activity (Baecke score).

${ }^{\mathrm{b}}$ Values are significant at $\mathrm{P}<0.05$.

The underlying pathophysiological mechanisms demonstrated the relationship between excess fat mass, obesity, and NAFLD include different points such as sys- temic inflammation, increased serum levels of free fatty acids, and subsequently insulin resistance (30).

Furthermore, the relationship between fatty liver con- 
Table 4. The Multivariate Linear Regression Analysis of the Association Between Liver Fat Content (\%) with BMD and BMC ${ }^{\text {a, b }}$

\begin{tabular}{|c|c|c|c|c|c|c|c|c|c|c|c|c|}
\hline \multirow{2}{*}{ Variables } & \multicolumn{3}{|c|}{ Model1 1} & \multicolumn{3}{|c|}{ Model 2} & \multicolumn{3}{|c|}{ Model 3} & \multicolumn{3}{|c|}{ Model 4} \\
\hline & $\mathrm{R}^{2}$ & в & P Value & $\mathrm{R}^{2}$ & $\beta$ & P Value & $\mathrm{R}^{2}$ & $\beta$ & P Value & $\mathrm{R}^{2}$ & $\beta$ & PValue \\
\hline Total BMD & 0.042 & 0.238 & 0.053 & 0.557 & -0.125 & 0.186 & 0.545 & -0.116 & 0.256 & 0.667 & -0.168 & 0.058 \\
\hline $\begin{array}{l}\text { Total BMD } \\
\text { Z-score }\end{array}$ & 0.027 & -0.205 & 0.094 & 0.096 & -0.217 & 0.105 & 0.079 & -0.263 & 0.07 & 0.111 & -0.285 & 0.048 \\
\hline Total BMC & 0.081 & 0.307 & 0.011 & 0.656 & -0.065 & 0.425 & 0.661 & -0.035 & 0.686 & 0.863 & -0.107 & 0.058 \\
\hline
\end{tabular}

Abbreviations: BMD, bone mineral density; BMC, bone mineral content; BMI, body mass index; ALT, alanine aminotransferase.

a Model 1, crude; Model 2, adjusted with age, BMI Z-score; Model 3, model 2 was additionally adjusted with ALT and fat mass index; Model 4, model 3 was additionally adjusted with total lean mass and physical activity (Baecke score).

$\mathrm{b}$ Values are significant at $\mathrm{P}<0.05$.

tent and Bone or osteoporosis-related indices was examined in the present study, and the findings revealed that liver fat accumulation was significantly correlated with lower whole-body BMD Z-score, regardless of total lean mass and physical activity. This finding suggests that liver steatosis may have a negative effect on bone health in obese children and adolescents. Consistent with our findings, some studies have confirmed the relationship between liver steatosis and BMD in children (14, 31-33). For example, Pirgon et al. documented that adolescents with obesity and NAFLD had lower lumbar spine BMD than adolescents with obesity and no NAFLD or non-obese participants (31). A recent study examining the relationship between hepatic fat content and BMD in children reported lower BMD and BMD Z-score in children with NAFLD (14). In contrast, Chang et al. evaluated vitamin D status and BMD in obese children with NAFLD and detected no difference in vitamin D levels or age-matched BMD among children with simple steatosis, NASH children, and healthy children (34). A systematic review and meta-analysis assessing the relationship between nonalcoholic fatty liver disease and bone mineral density in obese adolescents documented that obese adolescents with NAFLD had lower BMD and thus the higher risk of osteoporosis than obese children with no NAFLD (35).

The underlying pathophysiological mechanisms have not been clarified yet. However, several studies have suggested that chronic inflammation, exclusively produced by the liver, primarily increases CRP levels and elevated TNF- $\alpha$ levels in the systemic inflammation in this relationship (36-38). Furthermore, chronic inflammation can result in elevated osteoclast activity, inhibit osteoblast differentiation, and increase osteoblast apoptosis (39). In this regard, inflammatory cytokines, including interleukin-6, interleukin-1, and TNF- $\alpha$, have also been involved in the NAFLD pathogenesis (40). To conclude, the presence of systemic inflammation may justify the inverse relationship between NAFLD and total BMD Z-score.

Insulin resistance is another precise mechanism linking these two disorders. Moreover, the increased release of fat-free acids from adipocytes may lead to insulin re- sistance because of the extra accumulation of adipose tissue in hepatic mass (41), which is associated with BMD and bone health $(41,42)$. Further studies are recommended to address these mechanisms in detail.

The present study had some strengths. First, we excluded individuals with chronic conditions (e.g., viral hepatitis, autoimmune/congenital liver disease, congenital metabolic diseases, and cancer) who could yield CAP values for the different distribution of liver steatosis assessed by TE. Another strength of this study was that many possible intervening factors were included in the regression analyses.

This study also had some limitations. First, it was a cross-sectional study, making it difficult to detect a causal relationship between NAFLD with body composition and BMD. Further, liver biopsy as a gold standard approach to diagnosing and assessing liver steatosis and fibrosis was not conducted. According to recent studies, CAP and liver stiffness measurement (LSM) are well correlated with the degree of liver fibrosis and steatosis measured by biopsy $(43,44)$.

\subsection{Conclusion}

In conclusion, the present study results confirmed that excess adipose tissue is independently associated with higher NAFLD in obese and overweight adolescents. Moreover, it was demonstrated that liver steatosis is associated with decreased whole-body BMD Z-score, regardless of physical activity and lean mass. To sum up, these findings suggest that obesity and NAFLD may be closely correlated with bone loss and osteoporosis in adolescents.

\section{Acknowledgments}

We acknowledge the kind assistance and financial support provided by Mashhad University of Medical Sciences (MUMS).

\section{Footnotes}

Authors' Contribution: The authors' contribution was as follows: (1) Zahra Dehnavi, Farkhondeh Razmpour, Mah- 
moud Belghaisi Naseri, and Mohsen Ne'mati designed the research; (2) Zahra Dehnavi, Hanieh Barghchi, Fatemeh Roudi, Zahra Khorasanchi, and Andisheh Nourozian Ostad conducted the library search, collected the required data, and wrote the manuscript; (3) Zahra Dehnavi and Hanieh Barghchi tabulated the data. All of the authors read and approved the final manuscript.

Conflict of Interests: The authors declare that they have no competing interests to report.

Ethical Approval: The ethics committee approved the study protocol of this research of Mashhad University of Medical Sciences (MUMS) (Ethical Code: IR.MUMS.fm.REC.1395.64).

Funding/Support: Mashhad University of Medical Sciences financially supported this project.

Informed Consent: Written informed consent forms were provided by all the parents or legal guardians of children.

\section{References}

1. Feldstein AE, Patton-Ku D, Boutelle KN. Obesity, nutrition, and liver disease in children. Clin Liver Dis. 2014;18(1):219-31. doi: 10.1016/j.cld.2013.09.003. [PubMed: 24274876]. [PubMed Central: PMC4008146].

2. Cuda SE, Censani M. Pediatric obesity algorithm: A practical approach to obesity diagnosis and management. Front Pediatr. 2018;6:431. doi: 10.3389/fped.2018.00431. [PubMed: 30729102]. [PubMed Central: PMC6351475].

3. Khademi G, Nematy M, Ranjbar G, Pouryazdanpanah M, Rahimi R, Roudi F. Clinical nutrition approaches to medical management of children with obesity and critical illnesses.J Compr Ped.2019;11(1). doi: 10.5812/compreped.92108.

4. Pandita A, Sharma D, Pandita D, Pawar S, Tariq M, Kaul A. Childhood obesity: Prevention is better than cure. Diabetes Metab Syndr Obes. 2016;9:83-9. doi: 10.2147/DMSO.S90783. [PubMed: 27042133]. [PubMed Central: PMC4801195].

5. Welsh JA, Karpen S, Vos MB. Increasing prevalence of nonalcoholic fatty liver disease among United States adolescents, 1988-1994 to 2007-2010. J Pediatr. 2013;162(3):496-500 e1. doi: 10.1016/j.jpeds.2012.08.043. [PubMed: 23084707]. [PubMed Central: PMC3649872].

6. Takaki A, Kawai D, Yamamoto K. Molecular mechanisms and new treatment strategies for non-alcoholic steatohepatitis (NASH). Int J Mol Sci. 2014;15(5):7352-79. doi: 10.3390/ijms15057352. [PubMed: 24786095]. [PubMed Central: PMC4057677].

7. Angulo P. Nonalcoholic fatty liver disease. $N$ Engl $J$ Med. 2002;346(16):1221-31. doi: 10.1056/NEJMra011775. [PubMed: 11961152].

8. AlKhater SA. Paediatric non-alcoholic fatty liver disease: An overview. Obes Rev. 2015;16(5):393-405. doi: 10.1111/obr.12271. [PubMed: 25753407].

9. Soleimani D, Ranjbar G, Rezvani R, Goshayeshi L, Razmpour F, Nematy M. Dietary patterns in relation to hepatic fibrosis among patients with nonalcoholic fatty liver disease. Diabetes Metab Syndr Obes. 2019;12:315-24. doi: 10.2147/DMSO.S198744. [PubMed: 30881075]. [PubMed Central: PMC6420105].

10. Silveira LS, Monteiro PA, Antunes Bde M, Seraphim PM, Fernandes RA, Christofaro DG, et al. Intra-abdominal fat is related to metabolic syndrome and non-alcoholic fat liver disease in obese youth. BMCPediatr.
2013;13:115. doi: 10.1186/1471-2431-13-115. [PubMed: 23919592]. [PubMed Central: PMC3751250].

11. Briggs AM, Cross MJ, Hoy DG, Sanchez-Riera L, Blyth FM, Woolf AD, et al. Musculoskeletal health conditions represent a global threat to healthy aging: A report for the 2015 World Health Organization world report on ageing and health. Gerontologist. 2016;56 Suppl 2:S243-55. doi: 10.1093/geront/gnw002. [PubMed: 26994264].

12. Weaver CM. Parallels between nutrition and physical activity: Research questions in development of peak bone mass. Res Q Exerc Sport. 2015;86(2):103-6. doi: 10.1080/02701367.2015.1030810. [PubMed: 25965111].

13. Weaver CM, Gordon CM, Janz KF, Kalkwarf HJ, Lappe JM, Lewis R, et al. The National Osteoporosis Foundation's position statement on peak bone mass development and lifestyle factors: A systematic review and implementation recommendations. Osteoporos Int. 2016;27(4):1281386. doi: 10.1007/s00198-015-3440-3. [PubMed: 26856587]. [PubMed Central: PMC4791473].

14. Labayen I, Ruiz JR, Arenaza L, Medrano M, Tobalina I, Gracia-Marco L, et al. Hepatic fat content and bone mineral density in children with overweight/obesity. Pediatr Res. 2018;84(5):684-8. doi: 10.1038/s41390018-0129-2. [PubMed: 30120405].

15. Belghaisi-Naseri M, Dehnavi Z, Razmpour F, Tousi M, Taghipour A, Nematy $\mathrm{M}$, et al. Plasma levels of vascular endothelial growth factor and its soluble receptor in non-alcoholic fatty liver disease. J Nutrition Fasting Health. 2018;6(2):107-14.

16. Florindo AA, Latorre MRO. Validation and reliability of the Baecke questionnaire for the evaluation of habitual physical activity in adult men. Rev Bras de Medicina do Esporte. 2003;9(3):129-35. doi: 10.1590/s1517-86922003000300002.

17. Miller DJ, Freedson PS, Kline GM. Comparison of activity levels using the Caltrac accelerometer and five questionnaires. Med Sci Sports Exerc. 1994;26(3):376-82. [PubMed: 8183104].

18. Ono R, Hirata S, Yamada M, Nishiyama T, Kurosaka M, Tamura Y. Reliability and validity of the Baecke physical activity questionnaire in adult women with hip disorders. BMC Musculoskelet Disord. 2007;8:61. doi: 10.1186/1471-2474-8-61. [PubMed: 17610746]. [PubMed Central: PMC1931597].

19. Sadeghisani M, Dehghan Manshadi F, Azimi H, Montazeri A. validity and reliability of the Persian version of Baecke Habitual Physical Activity Questionnaire in healthy subjects. Asian J Sports Med. 2016;7(3). e31778. doi: 10.5812/asjsm.31778. [PubMed: 27826396]. [PubMed Central: PMC5098050].

20. Pathik P, Ravindra S, Ajay C, Prasad B, Jatin P, Prabha S. Fibroscan versus simple noninvasive screening tools in predicting fibrosis in highrisk nonalcoholic fatty liver disease patients from Western India. Ann Gastroenterol.2015;28(2):281-6. [PubMed: 25830783]. [PubMed Central: PMC4367221].

21. Sasso M, Miette V, Sandrin L, Beaugrand M. The controlled attenuation parameter (CAP): A novel tool for the non-invasive evaluation of steatosis using Fibroscan. Clin Res Hepatol Gastroenterol. 2012;36(1):1320. doi: 10.1016/j.clinre.2011.08.001. [PubMed: 21920839].

22. Gaia S, Carenzi S, Barilli AL, Bugianesi E, Smedile A, Brunello F, et al. Reliability of transient elastography for the detection of fibrosis in non-alcoholic fatty liver disease and chronic viral hepatitis.J Hepatol. 2011;54(1):64-71. doi: 10.1016/j.jhep.2010.06.022. [PubMed: 20932598].

23. Cummings-John OD, Geistweidt S, Okubote TC, Ascher Bartlett JM, Filo J, Betancourt A, et al. Fibroscan is a feasible and useful point-ofcare tool to assess nonalcoholic fatty liver disease severity in Children Texas Liver Institute, University of Texas (UT) Health San Antonio, San Antonio, TX. Am J Gastroenterol. 2019;114(1):S584-5. doi: 10.14309/01.ajg.0000593596.70904.a7.

24. Hsing JC, Nguyen MH, Yang B, Min Y, Han SS, Pung E, et al. Associations between body fat, muscle mass, and nonalcoholic fatty liver disease: A population-based study. Hepatol Commun. 2019;3(8):106172. doi: 10.1002/hep4.1392. [PubMed: 31388627]. [PubMed Central: PMC6671685]. 
25. Monteiro PA, Antunes Bde M, Silveira LS, Christofaro DG, Fernandes RA, Freitas Junior IF. Body composition variables as predictors of NAFLD by ultrasound in obese children and adolescents. BMC Pediatr. 2014;14:25. doi: 10.1186/1471-2431-14-25. [PubMed: 24476029]. [PubMed Central: PMC4016324].

26. Ramirez-Velez R, Izquierdo M, Correa-Bautista JE, Correa-Rodriguez M, Schmidt-RioValle J, Gonzalez-Jimenez E, et al. Liver fat content and body fat distribution in youths with excess adiposity. J Clin Med. 2018;7(12). doi: 10.3390/jcm7120528. [PubMed: 30544632]. [PubMed Central: PMC6306900].

27. Dehnavi Z, Ghandehari M, Razmpour F, Miryan M, Soleimani D, Parizadeh SM, et al. The associations of anthropometric parameters and hepatic steatosis. J Nutrition Fasting Health. 2020;8(1):23-7.

28. Razmpour F. Evaluating the accuracy and sensitivity of anthropometric and laboratory variables in diagnosing the liver steatosis and fibrosis in adolescents with non-alcoholic fatty liver disease.J Liver Res Disord Ther. 2018;4(3). doi:10.15406/jlrdt.2018.04.00114.

29. Yang HR, Chang EJ. Insulin resistance, body composition, and fat distribution in obese children with nonalcoholic fatty liver disease. Asia Pac J Clin Nutr. 2016;25(1):126-33. doi: 10.6133/apjcn.2016.25.1.15. [PubMed: 26965771].

30. Mazzotti A, Caletti MT, Sasdelli AS, Brodosi L, Marchesini G. Pathophysiology of nonalcoholic fatty liver disease: Lifestyle-gut-gene interaction. Dig Dis. 2016;34(Suppl 1):3-10. doi: 10.1159/000447275. [PubMed: 27548720].

31. Pirgon O, Bilgin H, Tolu I, Odabas D. Correlation of insulin sensitivity with bone mineral status in obese adolescents with nonalcoholic fatty liver disease. Clin Endocrinol. 2011;75(2):189-95. doi: 10.1111/j.13652265.2011.04038.x. [PubMed: 21521307].

32. Pacifico L, Bezzi M, Lombardo CV, Romaggioli S, Ferraro F, Bascetta S, et al. Adipokines and C-reactive protein in relation to bone mineralization in pediatric nonalcoholic fatty liver disease. World J Gastroenterol. 2013;19(25):4007-14. doi: 10.3748/wjg.v19.i25.4007. [PubMed: 23840146]. [PubMed Central: PMC3703188].

33. Pardee PE, Dunn W, Schwimmer JB. Non-alcoholic fatty liver disease is associated with low bone mineral density in obese children. Aliment Pharmacol Ther. 2012;35(2):248-54. doi: 10.1111/j.1365-2036.2011.04924.x. [PubMed: 22111971]. [PubMed Central: PMC4624396].

34. Chang EJ, Yi DY, Yang HR. Vitamin D status and bone mineral density in obese children with nonalcoholic fatty liver disease. J Korean Med Sci. 2015;30(12):1821-7. doi:10.3346/jkms.2015.30.12.1821. [PubMed: 26713058]. [PubMed Central: PMC4689827].

35. Sun Y, Dai W, Liang Y, Yang P, Yang Q, Liang M, et al. Relationship be- tween nonalcoholic fatty liver disease and bone mineral density in adolescents with obesity: A meta-analysis. Diabetes Metab Syndr Obes. 2019;12:199-207. doi: 10.2147/DMSO.S192256. [PubMed: 30787626]. [PubMed Central: PMC6363492].

36. Targher G, Lonardo A, Rossini M. Nonalcoholic fatty liver disease and decreased bone mineral density: Is there a link? J Endocrinol Invest. 2015;38(8):817-25. doi: 10.1007/s40618-015-0315-6. [PubMed: 26003827]

37. Ajmal MR, Yaccha M, Malik MA, Rabbani MU, Ahmad I, Isalm N, et al. Prevalence of nonalcoholic fatty liver disease (NAFLD) in patients of cardiovascular diseases and its association with hs-CRP and TNFalpha. Indian Heart J. 2014;66(6):574-9. doi: 10.1016/j.ihj.2014.08.006. [PubMed: 25634387]. [PubMed Central: PMC4310973].

38. Lucero D, Zago V, Lopez GI, Graffigna M, Fainboim H, Miksztowicz V et al. Pro-inflammatory and atherogenic circulating factors in nonalcoholic fatty liver disease associated to metabolic syndrome. Clin Chim Acta. 2011;412(1-2):143-7. doi: 10.1016/j.cca.2010.09.025. [PubMed: 20887718].

39. Bush H, Golabi P, Younossi ZM. Pediatric non-alcoholic fatty liver disease. Children. 2017;4(6). doi: 10.3390/children4060048. [PubMed: 28598410]. [PubMed Central: PMC5483623].

40. Cai D, Yuan M, Frantz DF, Melendez PA, Hansen L, Lee J, et al. Local and systemic insulin resistance resulting from hepatic activation of IKKbeta and NF-kappaB. Nat Med. 2005;11(2):183-90. doi: 10.1038/nm1166. [PubMed: 15685173]. [PubMed Central: PMC1440292].

41. Reid IR, Evans MC, Cooper GJ, Ames RW, Stapleton J. Circulating insulin levels are related to bone density in normal postmenopausal women. Am J Physiol. 1993;265(4 Pt 1):E655-9. doi: 10.1152/ajpendo.1993.265.4.E655. [PubMed: 8238341].

42. Stolk RP, Van Daele PL, Pols HA, Burger H, Hofman A, Birkenhager JC, et al. Hyperinsulinemia and bone mineral density in an elderly population: The rotterdam study. Bone. 1996;18(6):545-9. doi: 10.1016/8756 3282(96)00079-8. [PubMed: 8805995].

43. Sandrin L, Fourquet B, Hasquenoph JM, Yon S, Fournier C, Mal F, et al. Transient elastography: A new noninvasive method for assessment of hepatic fibrosis. Ultrasound Med Biol. 2003;29(12):1705-13. doi: 10.1016/j.ultrasmedbio.2003.07.001. [PubMed: 14698338].

44. Chon YE, Jung KS, Kim SU, Park JY, Park YN, Kim DY, et al. Controlled attenuation parameter (CAP) for detection of hepatic steatosis in patients with chronic liver diseases: a prospective study of a native Korean population. Liver Int. 2014;34(1):102-9. doi: 10.1111/liv.12282. [PubMed: 24028214]. 